\title{
PRÉSENTATION
}

Ce numéro de la Revue Internationale de Géomatique présente trois articles retenus suite à l'appel à communication «Innovations géomatiques pour les risques naturels et technologiques ». L'appel à communication avait été lancé suite à l'organisation d'un atelier sur cette thématique lors de la conférence SAGEO 2014 qui s'est tenue à Grenoble du 24 au 27 novembre 2014. En effet, l'information géographique est au cœur de la gestion des risques naturels et technologiques. Les récentes catastrophes ont montré la nécessité de disposer d'outils et de méthodes de plus en plus performants et innovants pour collecter, partager, combiner, fusionner, visualiser, analyser des données géographies multisources et hétérogènes. La gestion des risques naturels et technologiques doit s'appuyer sur la recherche en géomatique afin de trouver des solutions innovantes de gestion et de traitement de l'information géographique pour réduire l'impact des catastrophes sur la société et améliorer la prise de décision.

Les contributions ont fait l'objet d'une évaluation scientifique par un comité de lecture qui a relevé la qualité scientifique, l'originalité et le caractère innovant des travaux de recherche, plus particulièrement les innovations en géomatique.

Les articles proposent des travaux de recherche actuels qui mobilisent la géomatique afin d'améliorer la qualité de l'évaluation des risques naturels et technologiques. Les trois articles se focalisent sur une meilleure connaissance des enjeux en adoptant une approche vulnérabilité-centrée, conscients des limites des approches aléa-centrées face à la complexité des systèmes exposés aux phénomènes générateurs de risque. Ils partent également du constat qu'une évaluation plus fine des différentes composantes du risque, aléa et vulnérabilité, constitue la base pour le développement d'outils de prévention plus ciblés et ainsi plus efficaces. Ces travaux ouvrent vers des applications opérationnelles évidentes en matière de prévention des risques et de gestion des crises.

L'article de Mayaguezz et al. traite plus particulièrement de l'évaluation spatiotemporelle de l'exposition des populations face aux tsunamis à Padang (Indonésie). La méthode développée permet de connaître avec précision la population exposée aux tsunamis quels que soient le moment de la journée et la période de l'année. Grâce à des simulations tenant compte de l'accessibilité territoriale, un diagnostic des capacités d'évacuation est proposé.

L'article de Soto et Renard se focalise sur les vulnérabilités territoriales face aux aléas technologiques dans l'agglomération lyonnaise. Outre l'établissement d'un indice de vulnérabilité territoriale par analyse multicritère, dont les critères sont hiérarchisés 
par des experts suivant la méthode de l'Analytic Hierarchy Process, les auteurs proposent un croisement spatial entre aléas (représentés par différents types d'effets) et vulnérabilités des enjeux humains, matériels et environnementaux.

Enfin, l'article de Gonzva et al. porte sur l'évaluation des vulnérabilités des systèmes de transport guidé face aux inondations en mobilisant des méthodes issues de la sûreté de fonctionnement, qui s'intéressent plus particulièrement aux défaillances propres au système, et d'autres propres à la géomatique. En analysant en particulier la résilience des systèmes urbains, les auteurs proposent un modèle théorique solide avant de l'appliquer à un cas virtuel.

Paule-Annick Davoine

Elise BeCK

Université Grenoble-Alpes

\section{Comité de lecture}

Elise Beck - PACTE, Université Grenoble-Alpes

Emmanuel Bonnet - Résiliences, IRD

Alain Bouju - L3i, Université de La Rochelle

Sonia Chardonnel - PACTE, CNRS

Yves Cornet - Université de Liège, Belgique

Paule-Annick Davoine - LIG, Université Grenoble-Alpes

Jean-Paul Donnay - Université de Liège, Belgique

Thomas Devogele - LI, Université François Rabelais de Tours

Françoise Gourmelon - LETG, CNRS

Didier Graillot - École des Mines de Saint-Étienne

Thierry Joliveau - EVS, Université Jean-Monnet de Saint-Étienne

Didier Josselin - LIA, CNRS

Michel Lang - IRSTEA

Sylvie Lardon - AgroParisTech, INRA

Hélène Mathian - EVS, CNRS

Nathalie Molines - Université de Technologie de Compiègne

Aldo Napoli - Mines ParisTech, Paris

Eliane Propeck-Zimmermann - LIVE, Université de Strasbourg

Damienne Provitolo - Géoazur, CNRS

Anne Puissant - LIVE, Université de Strasbourg

Cyril Ray - Ecole Navale, Brest

Anne Ruas - IFSTTAR, Champs-sur-Marne

Lena Sanders - Géographie-cités, CNRS, Paris

Kamal Serrhini - CITERES, Université François Rabelais

David Sheeren - DYNAFOR, ENSAT, France

Christiane Weber - LIVE, CNRS 